\title{
Behavior of Motile Sperm in Taylor-Couette Flow: Effect of Shear Stress on the Behavior of Motile Sperm
}

\author{
Yasutaka Hayamizu ${ }^{1}$, Toru Hyakutake ${ }^{2}$, Koji Matsuura $^{3}$, Shinichiro Yanase ${ }^{4}$, \\ Shinichi Morita ${ }^{1}$, Shigeru Ohtsuka ${ }^{1}$, Takeshi Gonda ${ }^{1}$ \\ ${ }^{1}$ Department of Mechanical Engineering, Yonago National College of Technology, Tottori, Japan \\ ${ }^{2}$ Graduate School of Engineering, Yokohama National University, Yokohama, Japan \\ ${ }^{3}$ Research Core for Interdisciplinary Sciences, Okayama University, Okayama, Japan \\ ${ }^{4}$ Graduate School of Natural Science and Technology, Okayama University, Okayama, Japan \\ Email: hayamizu@yonago-k.ac.jp
}

Received May 27, 2013; revised June 4, 2013; accepted June 11, 2013

Copyright (C) 2013 Yasutaka Hayamizu et al. This is an open access article distributed under the Creative Commons Attribution License, which permits unrestricted use, distribution, and reproduction in any medium, provided the original work is properly cited.

\begin{abstract}
Infertility is often cited as one of the causes of a declining birthrate, which has become a serious social problem in recent years. Processes by which motile sperm can be safely and easily sorted are therefore important for infertility treatment. Therefore, as a new sorting method, microfluidic sperm sorter using the microfluidic system has been developed. To improve more separation efficiency of this device, it is necessary to know the behaviors of motile sperm in the microchannel where the sperm undergo shear flow. The previous study implied the necessity of the modeling of motile sperm in the shear flow. In the present study, therefore, we experimentally investigated the behavior of the motile sperm in the Taylor-Couette flow using PTV (Particle Tracking Velocimetry) method. The experimental results showed that the ascent of the shear stress led to the increase in the sperm velocity, and the direction of the sperm velocity was opposite to that of the flow.
\end{abstract}

Keywords: Motile Sperm; Taylor-Couette Flow; Shear Stress; PTV

\section{Introduction}

Infertility is often cited as one of the causes of a declining birthrate, which has become a serious social problem in recent years. Approximately $10 \%$ of couples were infertility, and almost $50 \%$ of all cases of infertility are associated with a lack of sperm or sperm abnormalities. Processes by which motile sperm can be safely and easily sorted are therefore important for infertility treatment. Currently, the swim up method or the Percoll method is employed for sperm sorting. However, these methods need much time and sort sperm in a non-physiological environment, so damage such as DNA fragmentation is a major problem [1], and it might result in the sorting of sperm that is unsuitable for fertilization [2]. Therefore, as a new sorting method, microfluidic sperm sorter using the microfluidic system has been developed (refer to Figure 1) [3-5]. To improve more separation efficiency of this device, it is necessary to know the behaviors of motile sperm in the microchannel where the sperm undergo shear flow. The previous study implied the necessity of the modeling of motile sperm in the shear flow.
In the present study, therefore, we experimentally investigated the behavior of the motile sperm in the Taylor-Couette flow using PTV (Particle Tracking Velocimetry) method. In this paper, we report the effect of shear stress on the behavior of motile sperm.

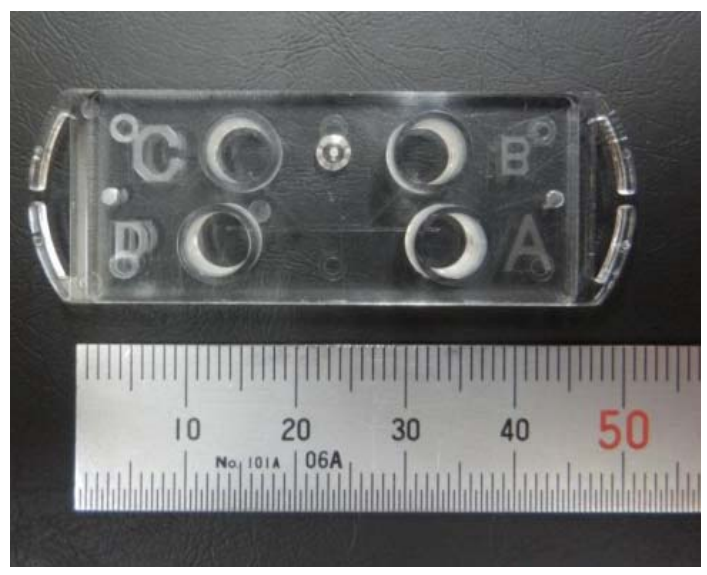

Figure 1. Microfluidic sperm sorter (Life Science Department, Menicon Co., Ltd.). 


\section{Experimental Procedure}

\subsection{Experimental Apparatus}

The dimension of the curved channel is shown in Table 1. Here, $a$ is the width of the curved channel (gap of the rotor and the casing), $h$ the height of the curved channel, $R$ the radius of the rotor (refer to Figures 2 and 3). The dimensionless parameter concerned is the Taylor number $\mathrm{Ta}$ [6] given by

$$
T a=\frac{R \omega a}{v} \sqrt{\frac{a}{R}},
$$

where $v$ is the kinematic viscosity and $\omega$ the angular velocity of the rotor. Taylor [6] classifies flows by the Taylor number $T a$ as follows:

$T a<41.3$ : Laminar Couette flow;

$41.3<T a<400$ : Laminar flow with the Taylor vortex.

We carried out an experiment by laminar Couette flow in $T a<41.3$ to examine the effect of shear stress on the behavior of motile sperm in this study.

We used bull sperm (AG Japan Co., Ltd.), which is

Table 1. Dimension of curved channel.

\begin{tabular}{ccc}
\hline$a[\mathrm{~mm}]$ & $h[\mathrm{~mm}]$ & $R[\mathrm{~mm}]$ \\
\hline 1 & 4 & 20 \\
\hline
\end{tabular}

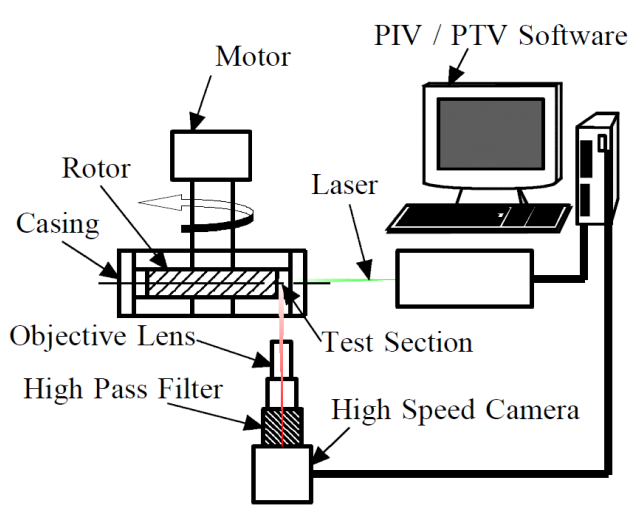

Figure 2. Experimental apparatus.

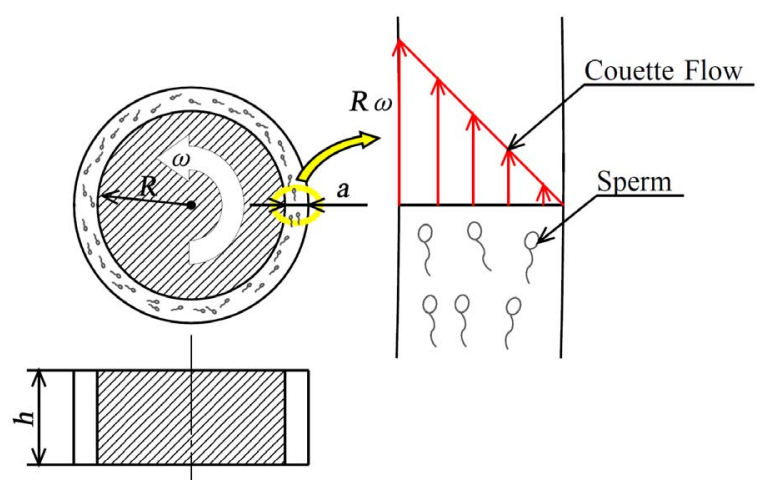

Figure 3. Enlargement of the test section. enclosed in a straw by $0.5 \mathrm{ml}$, and cryopreserved by liquid nitrogen $(77.15 \mathrm{~K})$. A saccharide, penicillin, citric acid for $\mathrm{pH}$ adjustment, and glycerin to prevent cell destruction in the fusion are added in the semen. A test for evaluate vitality of sperm is performed just after the extract and the fusion, and only sperms which meet a standard are enclosed in a straw, and they are cryopreserved again. Therefore, the motility of the sperm in the experiment is high.

\subsection{Experimental Method}

The working fluid mixed a fluorescent dye (Pyronin Y) and buffer solution (Modified HTF Medium) with semen. We diluted semen with the buffer solution, changed the viscosity of the working fluid and carried out an experiment. We used Pyronin Y as the fluorescence of sperms.

For an experiment procedure, we dissolved freeze semen in hot water of $310.15 \mathrm{~K}$ in 40 seconds. We scattered the fluorescent dyes in the buffer solution, and add it to sperms. Then, we filled the working fluid in the channel. The channel is formed of the casing and the rotor. The velocity gradient occurs between the rotor wall and the casing wall by rotating the rotor and thereby produces constant shear stress (refer to Figure 3). The rotor is rotated by the motor.

We next explain the method of visualization. The laser sheet lights up a cross-section of the channel normal to the channel side wall and the photographs are taken of the fluorescence patterns by a high speed camera. We used Davis7.2 (LaVision GmbH) for PTV and PIV, and used a fluorescent particle (FLUOSTAR: particle diameter $15 \mu \mathrm{m}$ ) of specific gravity 1.1 for a tracer of PIV. We calculated the sperm velocity by the PTV analysis of the fluorescence image of the sperm using the high speed camera with the high-pass filter $(570 \mathrm{~nm}$ or more transmitted wave length) (refer to Figure 4). Also, we calculated the flow velocity by tracing the PIV analysis of the fluorescent particles (refer to Figure 5).

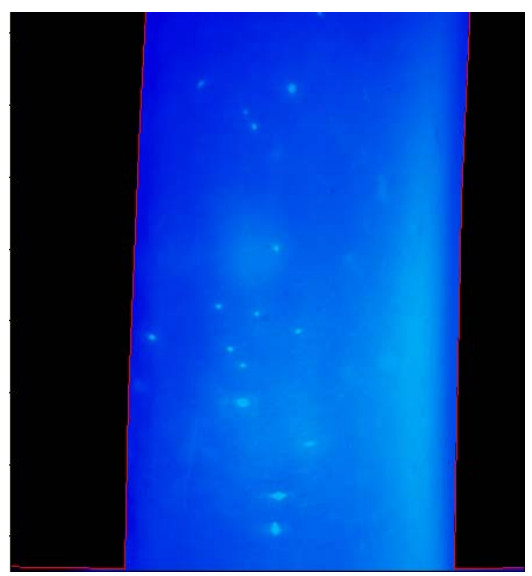

Figure 4. Fluorescence image of the sperm. 


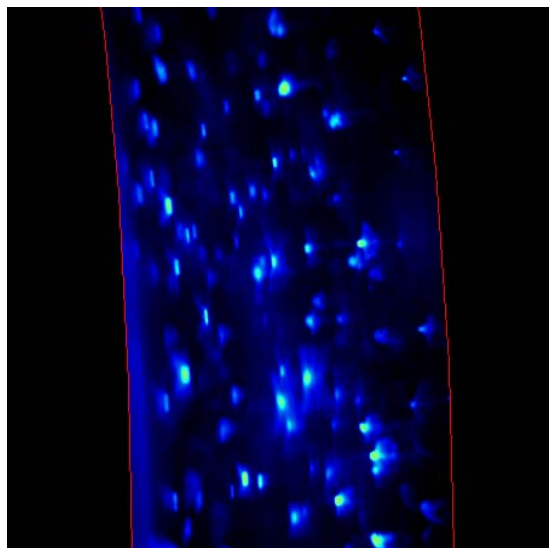

Figure 5. Fluorescence image of the particle.

We measured the viscosity of working fluid using the precision rotational viscometer before and after experiment and confirmed that there was not a viscosity change. Also, we calculated the density of the working fluid from the gravimeter.

\section{Experimental Results and Discussion}

\subsection{Physical Property}

Figures 6 and 7 show physical property of the working fluid at $310.15 \mathrm{~K}$. Figure 6 shows the relationship between the shear stress $\tau$ of each dilution rate $n$ and the shear velocity $\gamma$. The figure shows the relations of $\tau \propto \gamma$ in all dilution rates. This result means that the working fluid is Newtonian fluid even if dilution rate changes. In addition, a similar tendency was seen at other temperature.

Figure 7 shows the relationship between the viscosity $\mu$ and the dilution rate $n$. As the dilution rate of the working fluid increases, the viscosity decreases. Therefore, we carried out an experiment at dilution rate $n=2$ and 6 to examine the influence of the viscosity of the working fluid. The temperature of the working fluid is $310.15 \mathrm{~K}$ constant, which is suited for the sperm.

\subsection{Sperm Velocity}

Comparison between the experimental result and the theoretical result of the dimensionless flow velocity distribution in this present device is shown in Figure 8. The vertical axis of the figure is dimensionless flow velocity $u^{*}=u /(R \omega)$ and the horizontal axis is dimensionless coordinate $x / a$. The mean error rate of the experimental result to the theoretical result was approximately $9.3 \%$. Therefore, reliability of the measured values in this experiment is around $90.7 \%$.

The sperm velocity of $n=2$ and 6 is shown in Figure 9. The vertical axis of the figure is dimensionless sperm velocity $v^{*}=\left\{v(a / R)^{1 / 2}\right\} /(R \omega)$ and the horizontal axis is

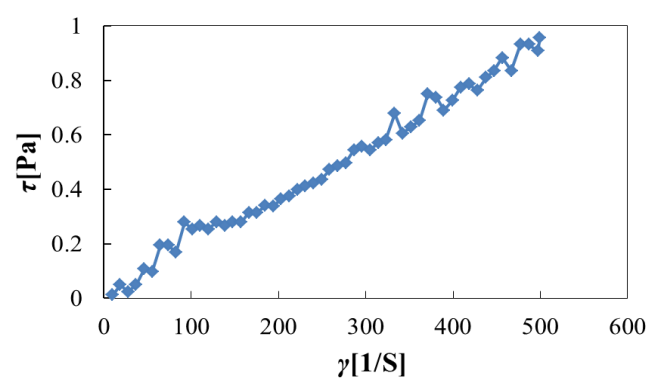

(a)

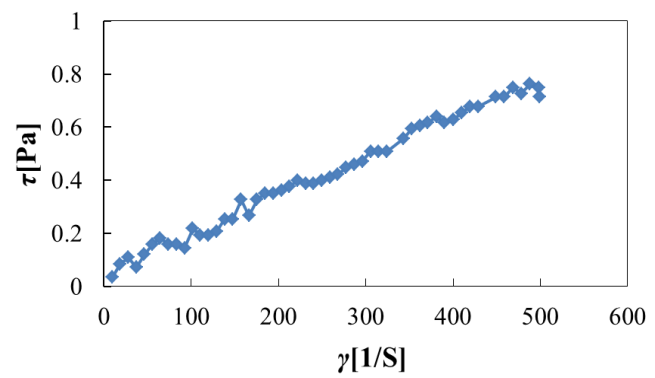

(b)

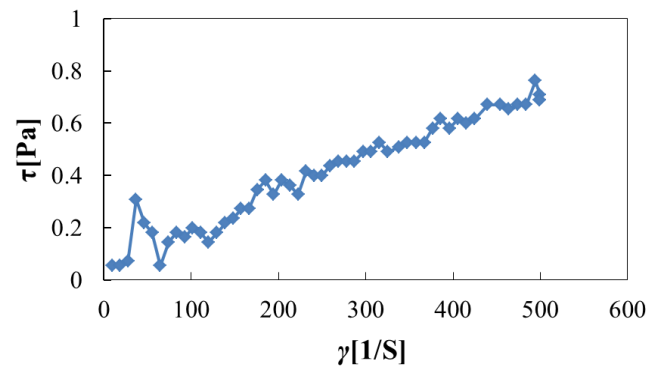

(c)

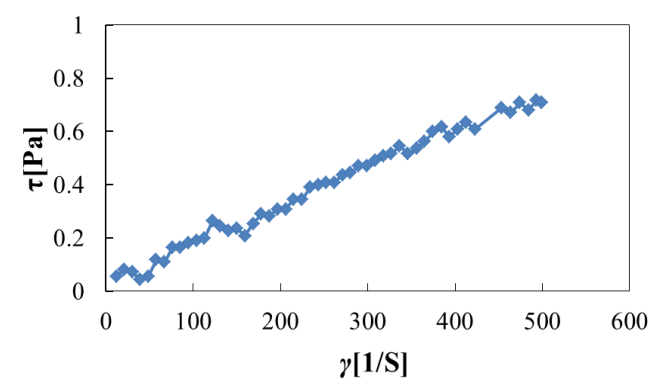

(d)

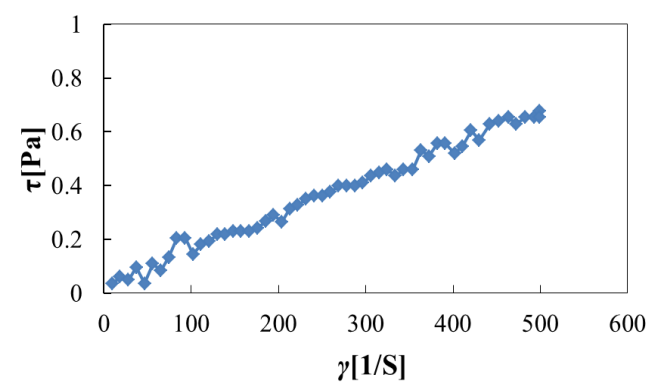

(e)

Figure 6. Relationship between $\tau$ and $\gamma$. (a) $n=2$; (b) $n=3$; (c) $n=4$; (d) $n=5$; (e) $n=6$. 


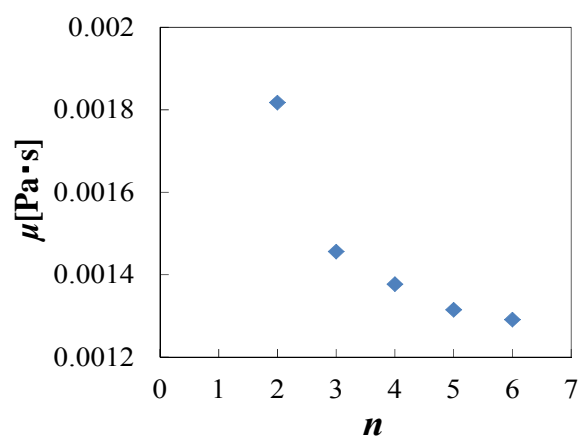

Figure 7. Relationship between $\boldsymbol{\mu}$ and $\boldsymbol{n}$.

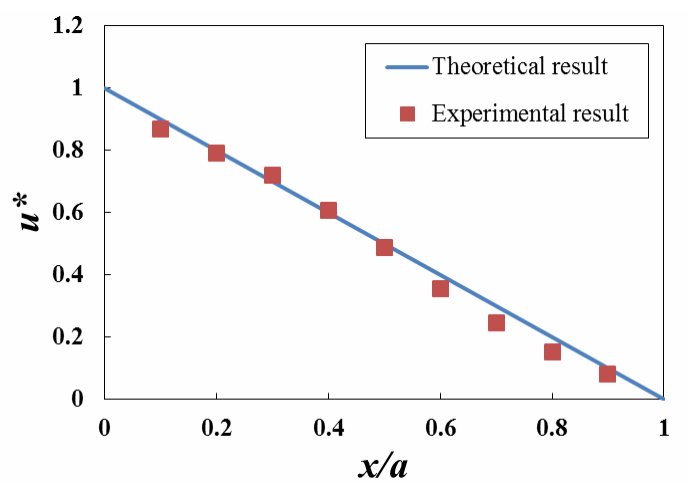

Figure 8. Velocity distribution of laminar Couette flow.

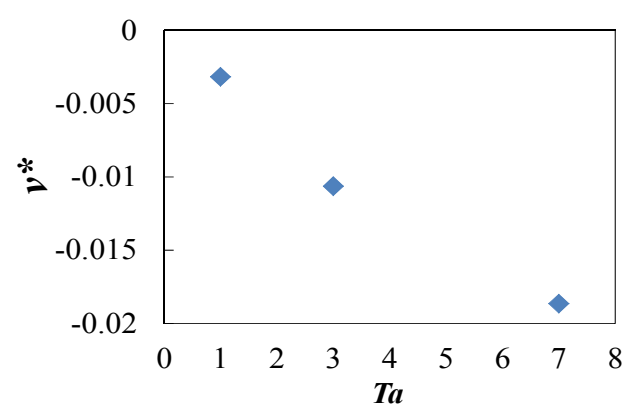

(a)

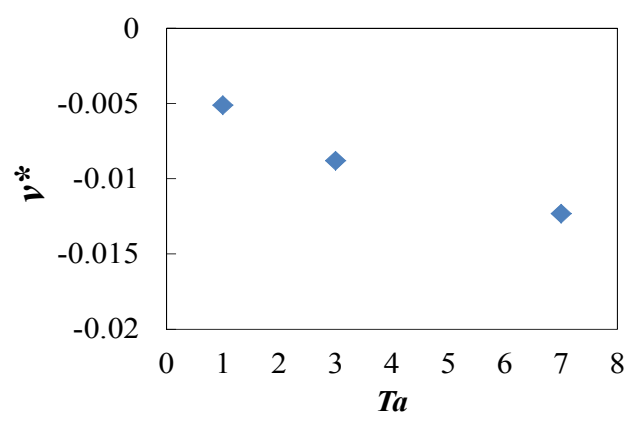

(b)

Figure 9. Sperm velocity in laminar Couette flow. (a) $n=2$; (b) $n=6$.

Taylor number $T a$. As Taylor number (shear stress) increases, the sperm velocity shows the tendency of increasing in all dilution rates. Then, the propulsion direc- tion of the sperm is opposite to the flow. In addition, in the difference of the sperm velocity is small in each $T a$ even if they compare it at dilution rate. In other words, even if the viscosity of working fluid is different, the sperm velocity shows not changing too much and agrees with the study of Smith et al. [7].

\section{Conclusions}

In the present paper, we experimentally investigated the behavior of the motile sperm in the Taylor-Couette flow using PTV method, and obtained following results:

The ascent of the shear stress led to the increase in the sperm velocity, and the direction of the sperm velocity is opposite to that of the flow.

Even if the viscosity of working fluid is different, the sperm velocity doesn't change too much.

\section{Acknowledgements}

The authors would like to express their cordial thanks to Hiroki Endo and Yuka Matsuda for their help in the experiments.

\section{REFERENCES}

[1] E. V. Younglai, D. Holt, P. Brown, A. Jurisicova and R. F. Casper, "Sperm Swim-Up Techniques and DNA Fragmentation," Human Reproduction, Vol. 16, No. 9, 2001, pp. 1950-1953. doi:10.1093/humrep/16.9.1950

[2] S. Smith, S. Hosid and L. Scott, "Use of Postseparation Sperm Parameters to Determine the Method of Choice for Sperm Preparation for Assisted Reproductive Technology," Fertility and Sterility, Vol. 63, No. 3, 1995, pp. 591-597.

[3] B. S. Cho, T. G. Schuster, X. Zhu, D. Chang, G. D. Smith and S. Takayama, "Passively Driven Integrated Microfluidic System for Separation of Motile Sperm," Analytical Chemistry, Vol. 75, No. 7, 2003, pp. 1671-1675. doi:10.1021/ac020579e

[4] T. G. Schuster, B. S. Cho, L. M. Keller, S. Takayama, and G. D. Smith, "Isolation of Motile Spermatozoa from Semen Samples Using Microfluidics," Reproductive BioMedicine, Vol. 7, No. 1, 2003, pp. 75-81. doi:10.1016/S1472-6483(10)61732-4

[5] T. Hyakutake, Y. Hashimoto, S. Yanase, K. Matsuura and K. Naruse, "Application of a Numerical Simulation to Improve the Separation Efficiency of a Sperm Sorter," Biomedical Microdevices, Vol. 11, No. 1, 2009, pp. 2533. doi:10.1007/s10544-008-9207-2

[6] H. Schlicting, "Boundary Layer Theory," 7th Edition, McGraw-Hill Pub. Co., New York, 1987, pp. 525-529,

[7] D. J. Smith, E. A. Gaffney, H. Gadelha, N. Kapur and J. C. Kirkman-Brown, "Bend Propagation in the Flagella of Migrating Human Sperm, and Its Modulation by Viscosity," Cell Motility and the Cytoskeleton, Vol. 66, No. 4, 2009, pp. 220-236. doi:10.1002/cm.20345 


\section{Nomenclature}

$a$ : width of the curved channel [mm] $h$ : height of the curved channel [mm] $n$ : dilution rate

$R$ : radius of the rotor [mm]

$T a$ : Taylor number $u$ : flow velocity $[\mathrm{mm} / \mathrm{s}]$

$u^{*}:$ dimensionless flow velocity $=u /(R \omega)$

$v$ : sperm velocity $[\mathrm{mm} / \mathrm{s}]$ $v^{*}:$ dimensionless sperm velocity $=\left\{v(a / R)^{1 / 2}\right\} /(R \omega)$ $x$ : distance from rotor wall [mm]

\section{Greek Letters}

$\gamma:$ shear velocity $[1 / \mathrm{s}]$

$\mu$ : viscosity $[\mathrm{Pa} \cdot \mathrm{s}]$

$v$ : kinematic viscosity $\left[\mathrm{mm}^{2} / \mathrm{s}\right]$

$\tau$ : shear stress $[\mathrm{Pa}]$

$\omega$ : angular velocity of the rotor $[\mathrm{rad} / \mathrm{s}]$ 